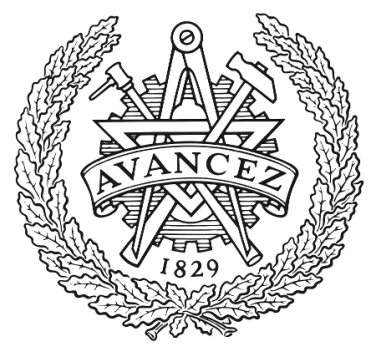

CHALMERS

UNIVERSITY OF TECHNOLOGY

\title{
Unsteady pressure analysis of the near wall flow downstream of the front wheel of a passenger car under yaw conditions
}

Downloaded from: https://research.chalmers.se, 2023-04-26 11:28 UTC

Citation for the original published paper (version of record):

Bonitz, S., Larsson, L., Sebben, S. (2018). Unsteady pressure analysis of the near wall flow downstream of the front wheel of a passenger car under yaw conditions. International Journal of Heat and Fluid Flow, 73: 188-198. http://dx.doi.org/10.1016/j.ijheatfluidflow.2018.07.007

N.B. When citing this work, cite the original published paper. 


\title{
Unsteady Pressure Analysis of the Near Wall Flow Downstream of the Front Wheel of a Passenger Car under Yaw Conditions
}

\author{
Sabine Bonitz \\ Dipl.-Ing. \\ Vehicle Engineering \& Autonomous \\ Systems \\ Department of \\ Mechanics and \\ Maritime Sciences \\ Chalmers University \\ of Technology \\ Gothenburg, Sweden \\ Email: \\ sabine.bonitz@chalmers.se \\ Lars Larsson \\ Professor \\ Marine Technology \\ Department of \\ Mechanics and \\ Maritime Sciences \\ Chalmers University \\ of Technology \\ Gothenburg, Sweden \\ Email: \\ lars.larsson@chalmers.se \\ Simone Sebben \\ Associate Professor \\ Vehicle Engineering \& Autonomous Systems \\ Department of Mechanics and Maritime Sciences \\ Chalmers University of Technology \\ Gothenburg, Sweden
}


general changes:

- structure changed: first results and discussion of the zero yaw, followed by results and discussion of the yaw configurations

\begin{abstract}
The flow around passenger cars is complex and characterized by many different structures and interactions. Occurring flow phenomena around a car determine crucial vehicle properties as driving stability, noise level and the aerodynamic performance. Despite they play also a significant role in terms of vehicle contamination. Therefore, it is of high importance to increase the understanding of the developing flow phenomena. Generic models are widely used to investigate flow structures and their interactions, but cannot serve to derive a general flow field for detailed full-scale vehicle models. A particularly complex area is the flow around the wheels and its interaction with the vehicle geometry. Studies on wheel-wheelhouse flow focus mainly on the geometrical influence of the wheel size, rim and tyre onto the aerodynamic drag and the flow field close to the wheel. The present work investigates the flow behind the front wheel arch of a full-scale passenger car. Time resolved surface pressure measurements were taken to study the near wall flow under different yaw conditions. Based on the data obtained, flow structures are identified and their propagation speed is calculated. Further, characteristic frequencies observed are discussed. It is found that coherent structures are present behind the front wheel arch, a larger one above wheel center height and smaller ones below it. These remain even under large yaw angles, no matter if the vehicle is yawed lee-or windward. The investigation further shows that two characteristic frequencies can be found, $S t=0.03$ and $S t=0.2$, whereby the latter is caused by the wheel rotation. The same frequencies occur also under yaw conditions, but are less pronounced when the measurement area is yawed to leeward.
\end{abstract}

\title{
Nomenclature
}

\section{Introduction}

The flow around passenger cars is a highly complex three dimensional bluff body flow which is characterized by vortices and recirculation regions of different sizes. The created flow structures in- 
teract with each other and determine crucial vehicle properties, as driving stability, noise level in the passenger compartment and the aerodynamic performance. But also regarding vehicle soiling it is important to understand the flow field. Apart from the customers demands not to touch dirty handles or to soil their clothes, safety aspects as driver visibility have to be considered. Especially, with the development of autonomous systems the usage of sensors and radars is increased. To ensure their functionality it is crucial to avoid their contamination.

To be able to understand this complex flow field, generic bodies as the Ahmed body or the SAE model are often used to study the development and impact of resulting flow structures; for instance in [1-4]. The study of simplified models allows to identify main features and to look into the impact of different effects, as the influence of edge radii. In the development if full vehicle geometries, the general findings are combined and the different flow phenomena interact with each other. Hence, it is also necessary to study detailed vehicle models. These further allow to consider details in the geometry and to study vehicle characteristic flow situations, as the flow through the engine bay and around the wheels $[5,6]$.

The flow around the wheels and through the wheelhouse receives high attention. This is mainly due to its special complexity. First the wheel is rotating in contact to the ground. Therefore, the simulation of the driving condition has to be considered. Second the wheel-vehicle interaction creates complex flow structures which are highly unsteady and sensitive to different parameters as for instance the rim design, tyre pattern or wheel size [7-13].

Simulating the driving conditions requires to include the wheel rotation and a moving ground representation. Automotive wind tunnels are therefore equipped with moving belt systems to rotate the wheels and to simulate a moving road. Wheel rotation adds another level of complexity. It is not enough to understand the flow around an isolated wheel, but also around rotating wheels in interaction with the vehicle. The wheel-vehicle interaction complicates the study, as flow phenomena cannot be isolated and studied individually.

Isolated wheel investigations were done as for instance by [7,13-16]. The flow topology for an isolated rotating wheel is for instance shown by Wäschle [7]. As a main structure a horseshoe vortex behind the wheel is reported. It is responsible for the main drag of the wheel and becomes weaker for the rotating wheel. On the upper side of the wheel a separation is created which has a ring vortex shape and causes an increase of pressure on top and behind the wheel reducing drag. The third observed structure is a 
vortex around the wheel shoulder at ground contact which is weaker compared to the horseshoe vortex. Schnepf et al. [13] explain on an isolated wheel the sensitive connection between tyre pattern and tyre load. Their results give insight in the significant changes of the wheel wake due to changes in these parameters.

Taking the wheel - wheelhouse and under body interaction into account, changes the topology around the wheel and its flow structure interactions [7, 15, 17-19]. Wäschle [7] shows the topology for a wheelwheelhouse configuration. Due to the wheelhouse the upper ring vortex described for the isolated wheel is not longer present. The horseshoe vortex created in the wheel wake and the vortices created at the lower shoulders are though still existent. At the rim flange a rim vortex appears. For a stationary configuration it has the shape of a horseshoe. Due to the wheel rotation the upper part of this horseshoe vortex is almost not existent. It is explained that for a stationary wheel the upper vortex trail is fed by the flow exiting the gap between wheel and wheelhouse. For a rotating wheel the mass flow exiting this gap is lower and therefore the upper vortex trail is not preserved. The lower vortex trail is fed by the flow through the rim and therefore dependent on the rim design. The only vortex which appears in the study purely due to wheel rotation is a longitudinal one appearing at the side, approximately at the wheel centre height. It is explained that this vortex is created due to the movement of the wheel wall against the flow direction. The rotation causes reverse flow on the upper wheel part and a separation bubble at the front side of the tyre. Due to the reverse flow a rotation is induced which causes the side vortex.

The flow physics downstream of the wheel arch are rarely investigated, especially its surface topology and surface pressure properties. In a numerical steady state investigation, done by Bonitz et. al. [20] the flow downstream of the front wheel and its development into the bulk flow is discussed based on surface streamlines (limiting streamlines) and 2D streamlines in cross planes, as well its surface pressure. It was discussed, how the limiting streamlines correlate with flow structures observable in the flow and how the surface properties can give insight to occurring flow phenomena.

Apart from the aerodynamic impact the created flow determines the level of contamination. Particles risen by the wheel rotation are transported downstream, stick to the vehicle side and accumulate. Gaylard \& Duncan [21] as well as Gaylard et al. [22] describe in their work the significant level of contamination onto the side of a SUV and notchback car due the wheel wake flow. In experimental and 
numerical investigations it is shown that the side soiling is mainly caused by flow being driven out the wheel house. Therefore the importance of an improved front wheel house design is emphasized.

However, in order to reduce side contamination, a better understanding of the flow physics in this area is needed to estimate the wheel wake dimension and to understand its behavior due to structures created by the wheel/ wheel house interaction under different conditions.

This work focuses on the near wall flow downstream of the front wheel of a fully detailed passenger car under different yaw conditions. By means of unsteady surface pressure measurements it is studied how different flow phenomena can be identified and how flow structures propagate downstream. The wheel-wheelhouse flow is altered by yawing the car lee- and windward, which shows how the flow structure dimensions and their propagation is changed. For all flow configurations the surface pressure, the pressure standard deviation and the dominant frequencies are analyzed. The study found coherent structures propagating downstream which remain even under large yaw angles. The spectral analysis showed that dominant frequencies can be found at $S t=0.2$ and $S t=0.03$, these are also present under yaw conditions, but appear to be less pronounced if the measurement location is yawed to leeward.

\section{Experimental Setup}

Unsteady surface pressure measurements were taken downstream of the front wheel of a full-scale passenger car. The experiments in the Volvo Cars aerodynamic wind tunnel in Gothenburg, Sweden. Figure 1 shows the vehicle in the wind tunnel environment.

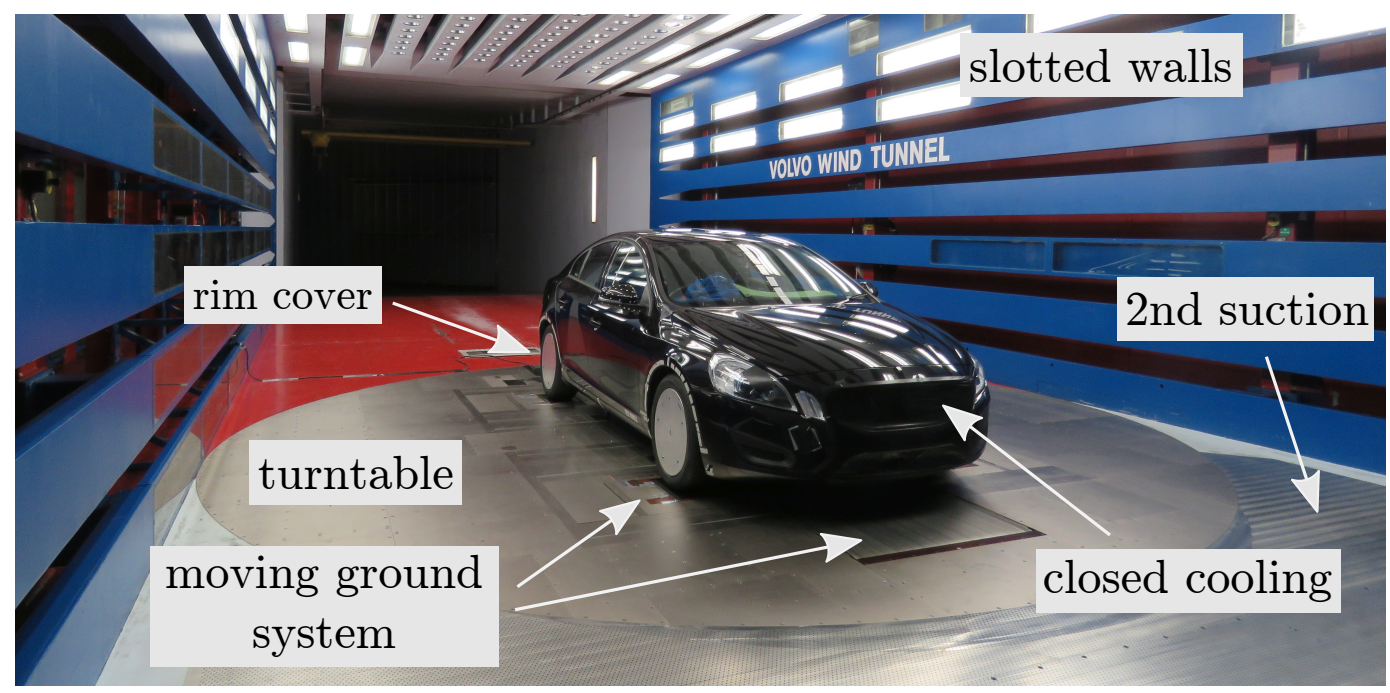

Fig. 1: Vehicle setup in the wind tunnel 


\subsection{Wind Tunnel}

The wind tunnel is of closed loop building type with slotted walls in the test section (30\% open area). The test section is $6.6 \mathrm{~m}$ wide, $15.8 \mathrm{~m}$ long and $4.1 \mathrm{~m}$ high, which leads to a cross sectional area of $27.06 \mathrm{~m}^{2}$. The test object is fixed in its place by four rigid struts. To simulate the moving road, a boundary layer control systems and a five belt system are installed (see Figure 1). The wheels are rotated by spinning the belts underneath each wheel (called wheel drive units). The installed turntable allows to yaw the vehicle up to $\pm 30^{\circ}$. During the experiment configurations at $\beta=0^{\circ}, \beta= \pm 5^{\circ}, \beta= \pm 10^{\circ}$ and $\beta= \pm 15^{\circ}$ were tested. Figure 2 shows a schematic of the wind tunnel setup and its coordinate systems. The flow is driven by a $5 \mathrm{MW}$ fan and allows test speeds up to $250 \mathrm{~km} / \mathrm{h}$. The test speed in the experiment was $100 \mathrm{~km} / \mathrm{h}$, which corresponds to a Reynolds number of $R e=6.1 \cdot 10^{6}$ based on the vehicle length $(l=4.63 \mathrm{~m})$. The wind tunnel nozzle contraction ratio is $6: 1$ and the turbulence level at the center of the turntable is $0.1 \%$. The blockage due to the car frontal area is $8 \%$ at zero yaw. A more detailed description about the wind tunnel and its road simulation can be found in [23].

\subsection{Test Object}

The test object was a full-scale Volvo S60 production car of notchback type (model year 2010). For the experiments the cooling inlets in the front of the car were closed to exclude the influence from the engine bay flow. Additionally the rims were covered with an aluminum plate, to reduce rim effects, see also Figure 1. The rims were 17 inch and the tyre size was 215/50R17. The vehicle has a length of $4.63 \mathrm{~m}$ and a frontal area of $2.27 \mathrm{~m}^{2}$. The production suspension was replaced by a rigid suspension to

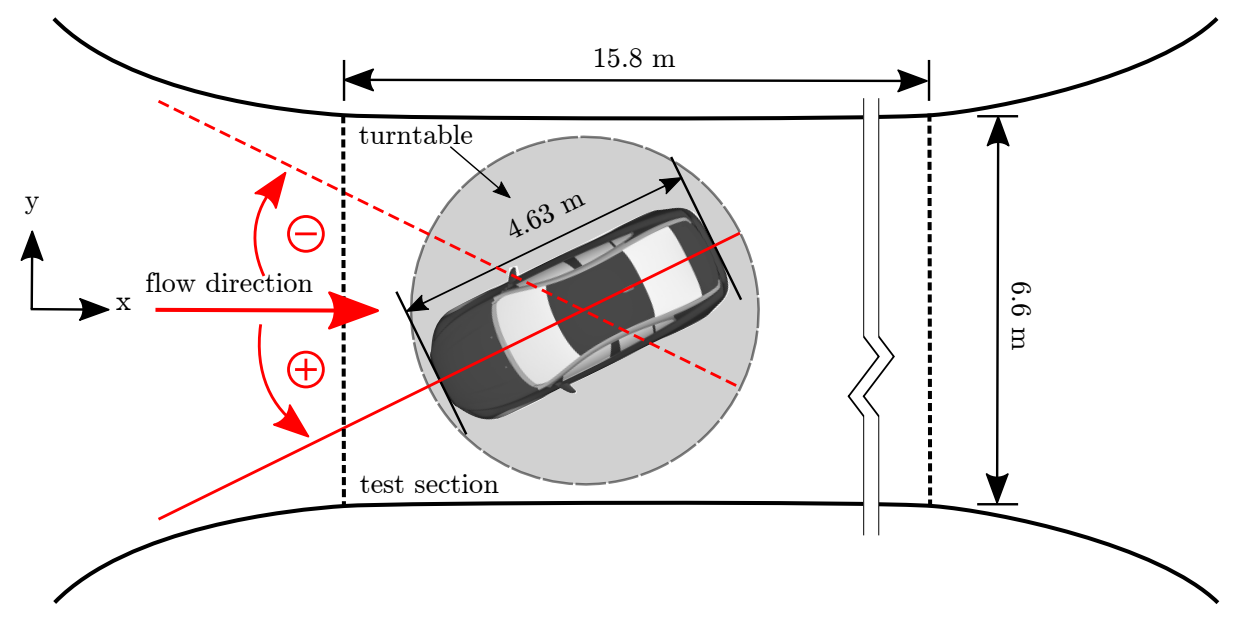

Fig. 2: Schematic wind tunnel setup and coordinate system 
keep the wheel center in a fixed position. This avoids vertical movements of the wheel due to radial expansion of the tyres.

\subsection{Pressure Measurements}

For the surface pressure measurements, unsteady pressure probes from First Sensor - Sensor Techniques (HCLA0025DB) were connected through drilled holes. Through tubes with a length of less than $50 \mathrm{~mm}$ and a diameter of $1.5 \mathrm{~mm}$ the sensors were attached to the measurement location. This setup ensures a smooth surface and no disturbances onto the near wall flow. The sensors have a range of $\pm 2500 \mathrm{~Pa}$ and an accuracy of $\pm 5 \mathrm{~Pa}$. The sensitivity is about $0.0007999 \mathrm{~V} / \mathrm{Pa}$ and the uncertainty is $5.2 \times 10^{-7} \mathrm{~V} / \mathrm{Pa}$. The used tubing length is within a range were no transfer function correction is needed. Pressure measurements in the empty tunnel did not show any characteristic frequencies which can be attributed to the tunnel geometry and flow. The location of the sensors and their individual identification is shown in Figure 3.

At a sampling frequency of $1000 \mathrm{~Hz}$, it was possible to take measurements for 15 sensors simultaneously. Therefore the area was split into two sections (sensors 1 to 15 and sensors 16-30). During the measurement, data was recorded over $10 \mathrm{~min}$, while the frequency range of interest was below $100 \mathrm{~Hz}$. For yaw configurations, measurements at a sampling frequency of $200 \mathrm{~Hz}$ over 1 min were taken. The reduced sampling frequency allowed to measure in 40 points simultaneously, to capture the flow situa-

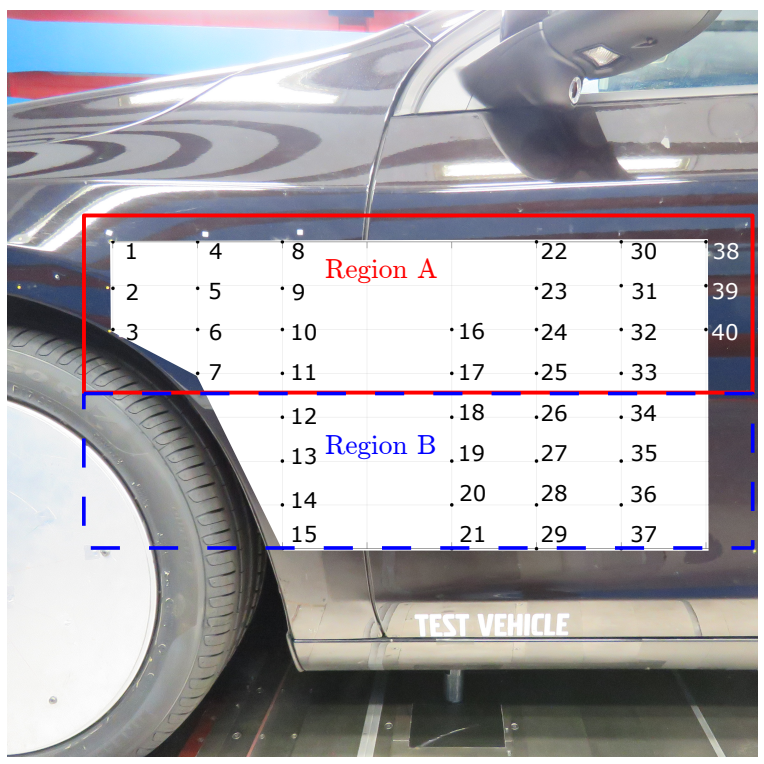

Fig. 3: Measurement grid and individual identification of the pressure sensors 
tion created over a larger area due to larger yaw angles. The reference pressure pressure is taken at the wind tunnel nozzle, which was also the wind tunnel reference pressure and the pressure data was not corrected.

\section{Results and discussion}

The following sections present the results in terms of pressure distribution, frequency analysis and cross correlations. First the baseline case at yaw angle $\beta=0^{\circ}$ is presented which is then followed by the results for the different yaw configurations. The discussion of the results follows in a separate section.

\subsection{Results of the zero yaw case}

\subsubsection{Surface Pressure}

The time averaged pressure distribution is presented in Figure 4 in terms of the dimensionless pressure coefficient. Along the upper wheelhouse edge a low pressure area (1) can be found, while a pressure increase is observed along the lower wheelhouse edge (2). The area directly downstream of the wheelhouse edge is characterized by a lower pressure (3), which recovers moving downstream (4). This pressure distribution shows that the flow around the wheel and through the wheelhouse create a separation which results in the observed low pressure (3). However, from the distribution it is not possible to identify if the created separation is of recirculation (bubble type) or of a vortex type.

The standard deviation of the pressure coefficient is shown in Figure 5. The highest fluctuations can be found at the upper wheel arch edge extending horizontally downstream with a clear spike as shown in region (1). It is assumed that the fluctuations are created by the flow exiting the wheelhouse and reattaching in this area. It is also expected to observe a separation along the lower wheelhouse edge (2). However, the pressure at the lower edge is higher than the pressure around the upper area where a separation is supposed and also the fluctuations are very low in this area. Hence, the separation at the edge created is very weak compared to the separation in (1).

\subsubsection{Frequency spectrum}

The pressure data is also analysed regarding its characteristic frequencies, which can be related to occurring flow phenomena. The frequency spectrum showed dominant frequencies in all signals. Figure 6 shows exemplary the spectrum for sensors 5 and 24 . For the investigated flow condition, several 


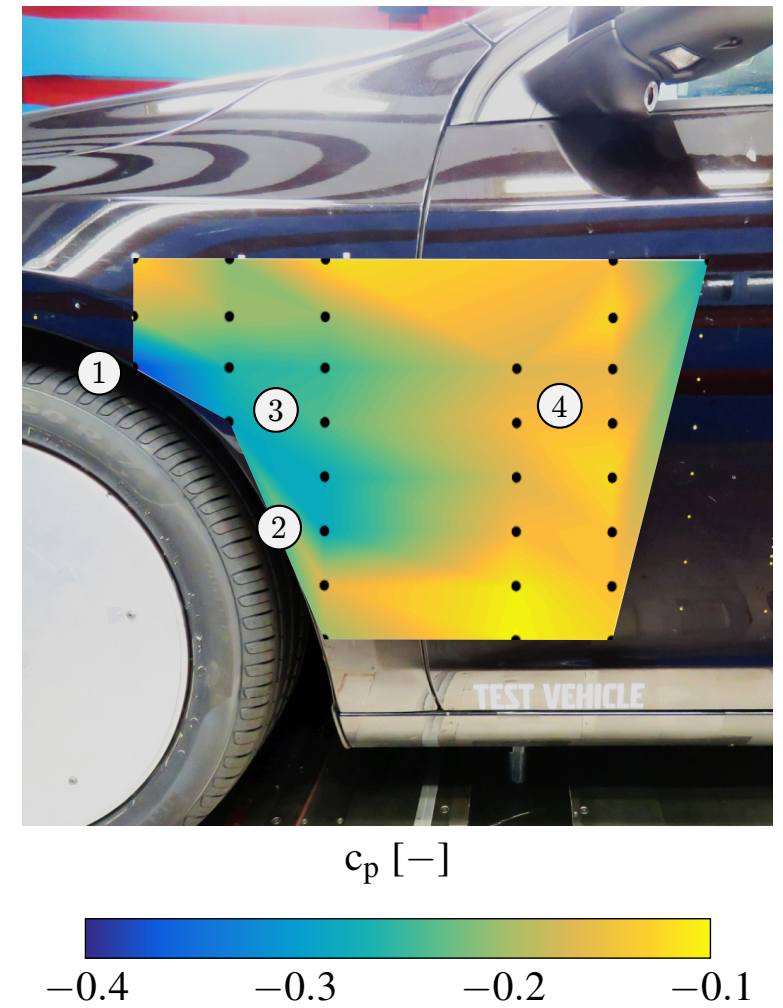

Fig. 4: Measured pressure distribution behind the front wheel arch

dominant frequencies can be found. The most dominant peaks are shown at $\mathrm{f}=13.9 \mathrm{~Hz}, \mathrm{f}=27.8 \mathrm{~Hz}$, $f=41.7 \mathrm{~Hz}, \mathrm{f}=55.6 \mathrm{~Hz}$ and $\mathrm{f}=69.5 \mathrm{~Hz}$. These are all harmonics of the base frequency $\mathrm{f}=13.9 \mathrm{~Hz}$. Calculating the Strouhal number

$$
\mathrm{St}=\frac{f l}{u}
$$

based on the rim diameter $l_{\text {rim }}=0.43 \mathrm{~m}$ and the inlet velocity $\mathrm{u}=27.78 \mathrm{~m} / \mathrm{s}$, gives $\mathrm{St}=0.22$. Using the wheel diameter $l_{\text {wheel }}=0.54 \mathrm{~m}$ as a characteristic length gives $\mathrm{St}=0.27$. A closer look into the lower spectrum shows that a further frequency can be identified at $f=1.9 \mathrm{~Hz}$, which corresponds to St $=0.03$ based on the rim diameter. The frequency of $f=13.9 \mathrm{~Hz}$ corresponds also to the rotational frequency of the wheel. In a configuration with stationary wheels, it was observed that the $13.9 \mathrm{~Hz}$ peak and its harmonics diminish (Figure 7). It is therefore assumed that the wheel rotation causes the occurrence of the $13.9 \mathrm{~Hz}$ peak. However, the peak at $f=1.9 \mathrm{~Hz}$ is still present. 


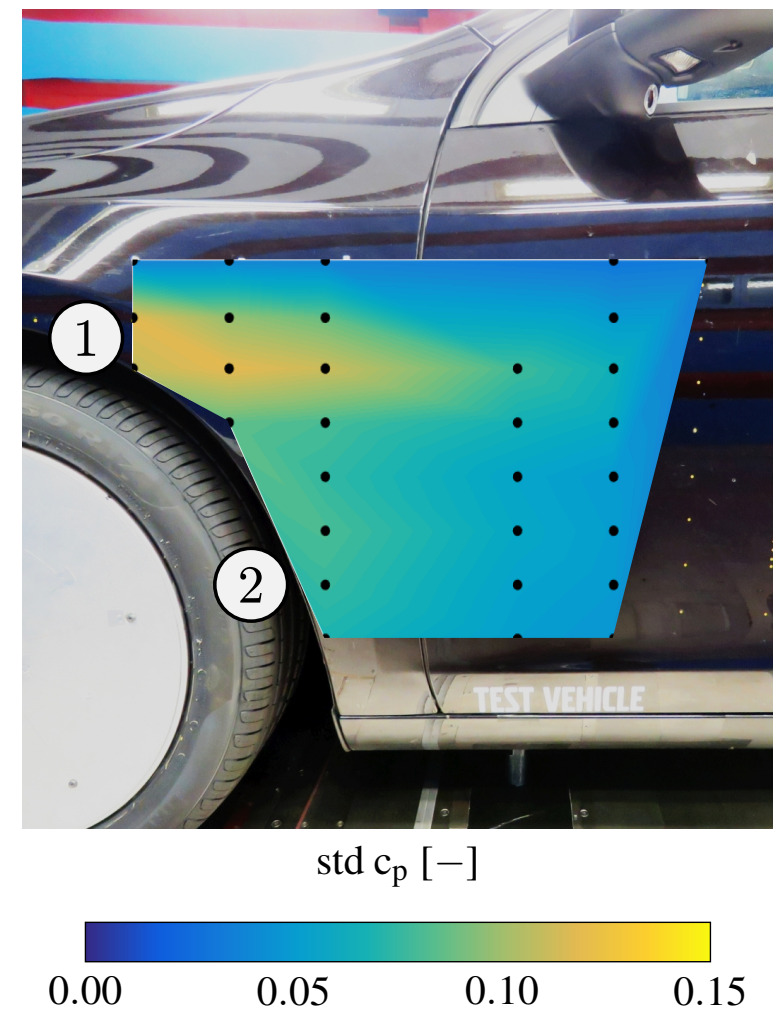

Fig. 5: Standard deviation of the pressure coefficient

\subsubsection{Cross correlations}

Figure 8 shows the cross correlation coefficient distribution regarding the signal of sensor 2. It can be seen that signal 2 has similarities with the signals in the upper half (region A), but no correlation with the lower signals (signal 12 to 15 ). This leads to the conclusion, that the flow condition in region A is independent from what happens in region B (visualized by Figure 8). Looking into the individual cross correlation signals, shown in Figure 9, confirms the connection among the sensors in region A, while the signal does not show similarities with sensor 12 .

The propagation of the structure, starting at location 2, can also be calculated by means of the correlation signals respective signal 2 (Figure 9). Measurement location 1 an 3 are are located at the same vertical line as sensor 2. The signals show similarities, but no shift in time. Hence, the structure reaches over all three measurement locations. Downstream, the highest correlations can be found with signal 5,6 and 10. These are also shifted in time, which shows a downstream movement of the structure. Based on the time shift, the propagation speed can be calculated. The values are summarized in Table 1. The main propagation is in horizontal direction with a slight downwash, moving approximately with $1 / 2$ of the inlet velocity. 


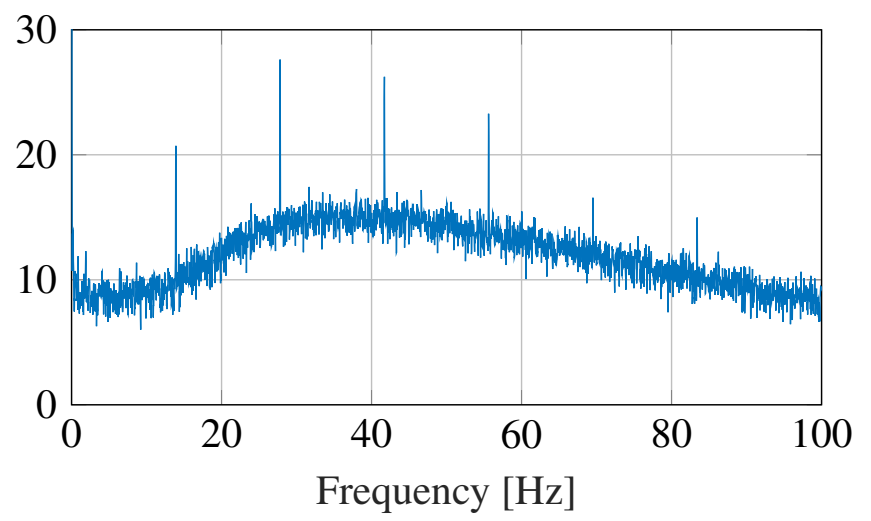

(a) frequency spectrum sensor 5

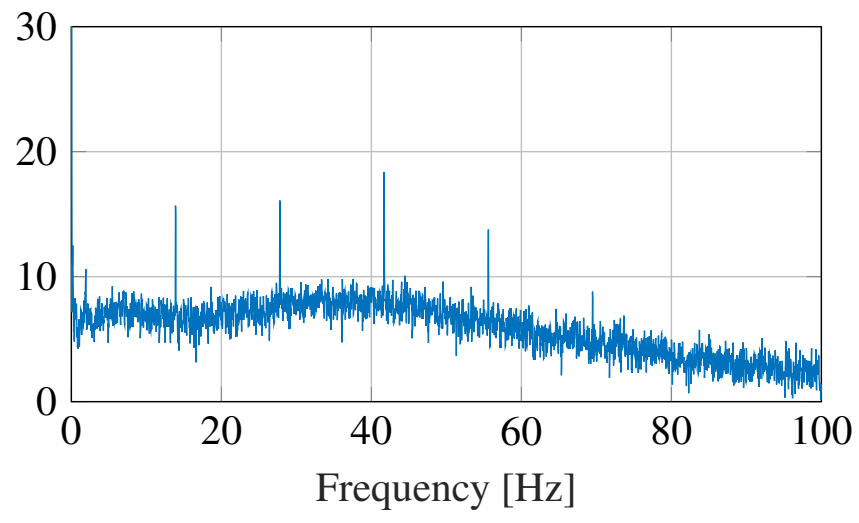

(b) frequency spectrum sensor 24

Fig. 6: Exemplary frequency spectrum for sensor 5 and 24

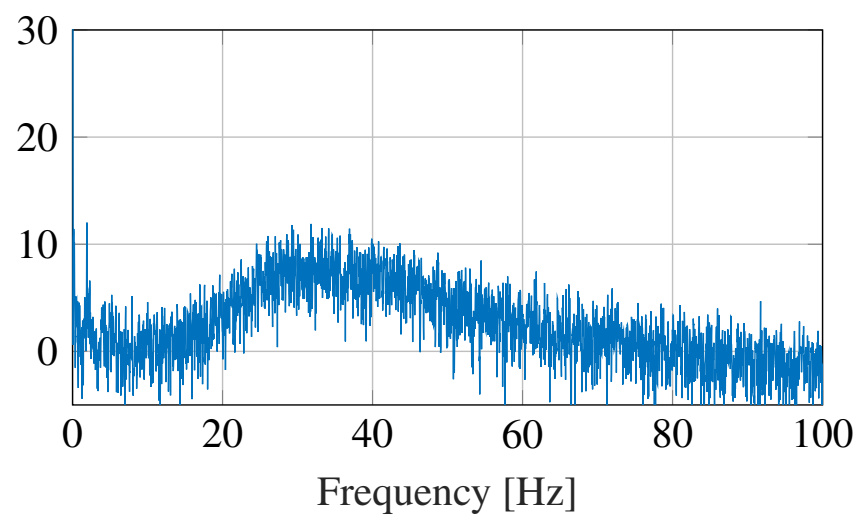

Fig. 7: Frequency Spectrum of sensor 24 for stationary wheels

Weaker correlations are found with signal 4, 7, 8, 9 and 11. As discussed before there is no correlation with signals 12 to 15 . This implies that over the investigated area one large coherent structure can be observed in the upper part, which is independent from the lower part. Comparing the cross correlation distribution and signals of Figure 8 and 9 with the standard deviation of the surface pressure, it is observable that the correlations extend over the area where also the high pressure fluctuations occur. 


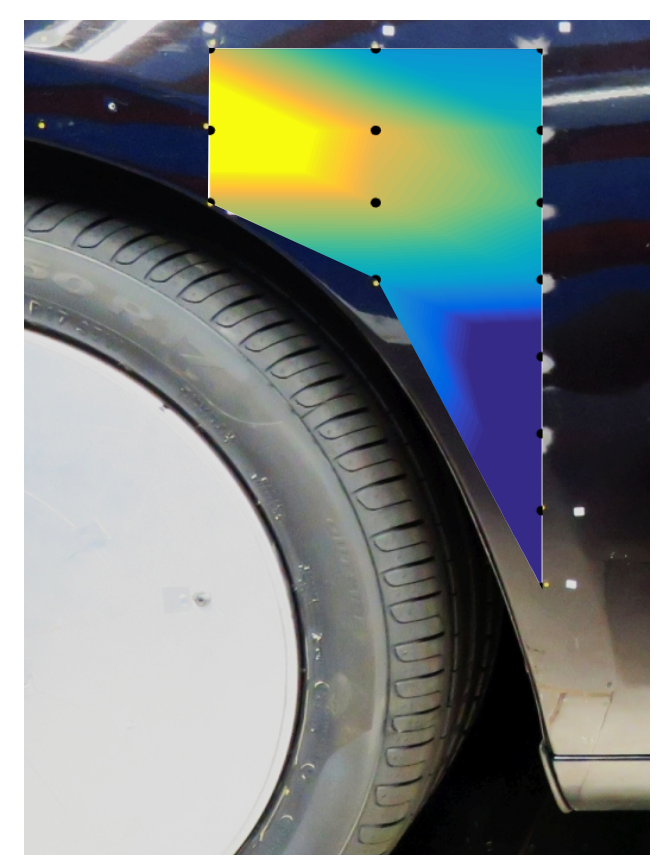

cross correlation coefficient

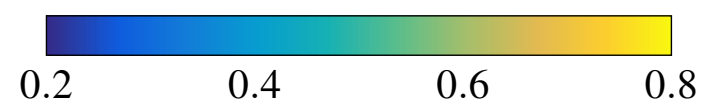

Fig. 8: Cross correlation regarding signal 2

Table 1: Propagation speed based on cross correlation time shift

\begin{tabular}{|l|c|c|c|}
\hline Sensors & corr coeff & $\tau[\mathrm{ms}]$ & propagation speed [m/s] \\
\hline \hline S2/S5 & 0,677 & 9 & 11,11 \\
\hline S2/S6 & 0,637 & 10 & 11,18 \\
\hline S2/S7 & 0,420 & 10 & 14,14 \\
\hline S2/S8 & 0,372 & 12 & 17,18 \\
\hline S2/S9 & 0,465 & 14 & 14,29 \\
\hline S2/S10 & 0,516 & 16 & 12,88 \\
\hline S2/S11 & 0,389 & 16 & 13,98 \\
\hline
\end{tabular}

Figure 10a shows the cross correlation signals between S13/S14, S14/S15 and S13/S15. The correlation signal between S13/S14 and S14/S15 show weaker correlations compared to the signals from the top region. Between S13 and S15 no significant correlation (lower than 0.2) can be found. From the correlations in the lower part it can be seen, that there is no large coherent flow structure created which reaches over all of the lower sensors. Selected cross correlation signals downstream of sensors 13 to 

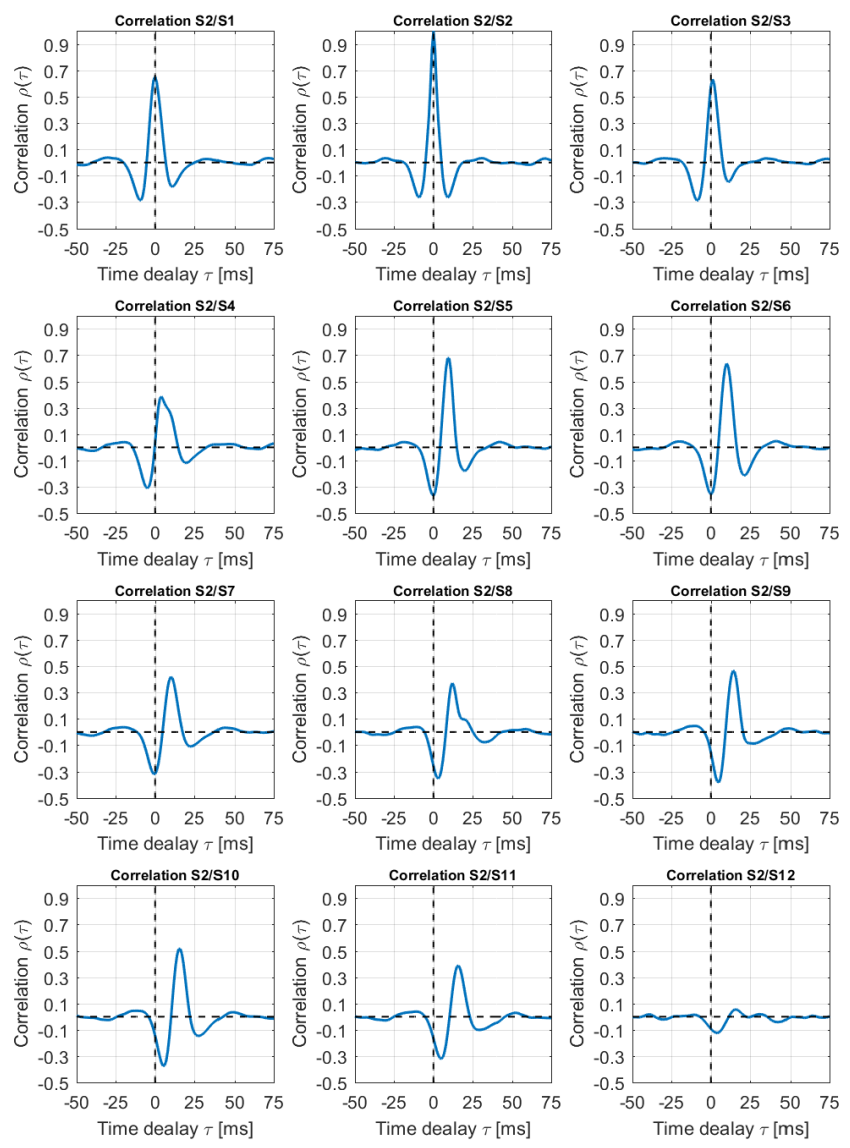

Fig. 9: Cross correlation signals respective sensor 2

15 are shown in Figure 10b. The analysis shows that the highest correlations are found in horizontal direction (S20/S28). Between S20/S27 and S20/29 (diagonal downstream directions) the correlations are weaker and the comparison between S29 and S27 show no significant correlation. Therefore it can be suggested, that no large enough flow structure is present, to reach over different sensors (in vertical direction). It rather shows that the flow is moving almost undisturbed downstream in mean flow direction.

\subsection{Discussion of the zero yaw configuration}

The pressure distribution in the area downstream of the front wheel house showed a lower pressure in the upper area, directly behind the wheel arch edge (Figure 4). From the pressure standard deviation (Figure 5) it can be concluded that the area with the occurring spike (Figure 5, (1)) corresponds to the reattachment area of a flow structure exiting the wheel house. The cross correlations in Figure 8 and 9 also showed a coherent structure in this region. Downstream, this structure aligns with the mean flow direction, as the correlations between neighboring sensors becomes weaker. For the region B in the 

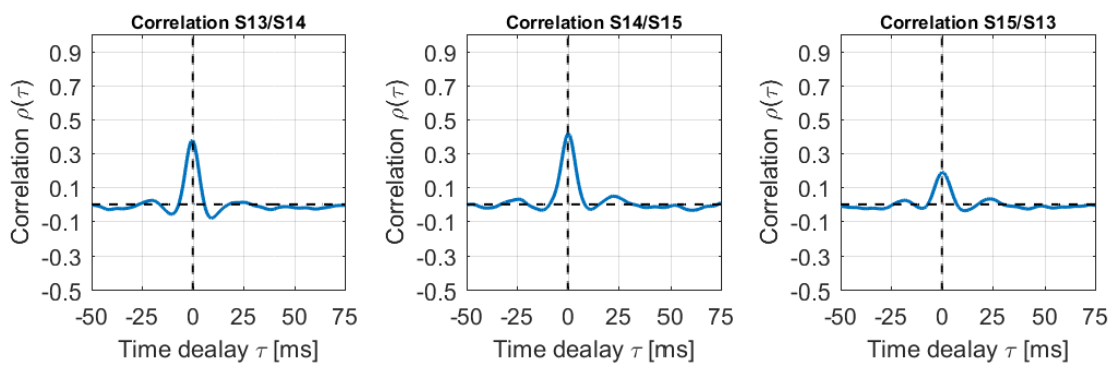

(a)
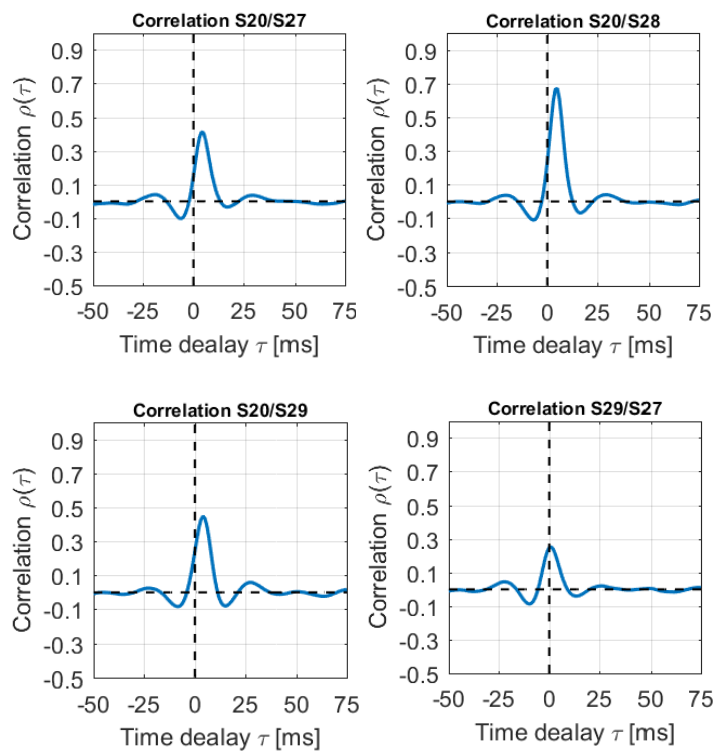

(b)

Fig. 10: Cross correlations for selected sensors in region B

investigated area, the correlations indicate a flow aligned with the mean flow direction throughout the investigated distance (Figure 10). As the pressure distribution showed a higher pressure compared to the region behind the upper wheelhouse edge and only weak fluctuations were observed by the standard deviation, it can be assumed that the flow is attached all the way. This means that the flow separating at the lower wheelhouse edge has a very short reattachment length. A reason for this might be that the closed rim and the flow around the front of the car create a fairly smooth flow with high momentum, which is not disturbed by flow exiting the wheelhouse.

A reason for the flow exiting the wheelhouse in the upper part might be an interplay between the wheel rotation and the oncoming flow entering the wheelhouse. The flow entering the wheelhouse (mainly from the underbody) points in $\mathrm{x}$ direction, while the wheel rotation drags flow up into the wheelhouse. The combination of these effects, pushes fluid out from the wheelhouse, creating a separation structure, which seems to be vortex like and exits the wheelhouse in the observed region. 
The analysis of the frequency spectrum showed a dominant frequency of $\mathrm{St}=0.2$. This is a characteristic value found for different separation and vortex shedding phenomena. It was shown that this frequency is only present for rotating wheels. A second characteristic frequency could be found at $\mathrm{St}=0.03$. Contrary to the previous one this is also present in a stationary wheel configuration. Wieser et al. [24] investigated the flow behavior around a DrivAer scale model, which is also a notchback shape as the here investigated car. Among others unsteady pressure measurements were taken downstream of the rear wheelhouse. In this region they found a characteristic frequency of $\mathrm{St}=0.28$ based on the vehicle length. Calculating the Strouhal number for the $1.9 \mathrm{~Hz}$ peak based on the vehicle length $\left(l_{\mathrm{VL}}=4.63 \mathrm{~m}\right)$ gives $\mathrm{St}_{\mathrm{VL}}=0.32$. It can be assumed that in both cases similar phenomena were observed. Wieser et al. did not identify any further dominant frequencies, especially none in the order of $\mathrm{St}=0.2$ for the rear wheel region, but as it was shown $\mathrm{St}=0.2$ is related to the wheel rotation, while Wieser et al. measured with stationary wheels. This emphasizes the suggestion that the flow interaction with the wheelhouse creates frequencies which are observable for stationary and rotating wheel configurations.

In an investigation done by Bonitz et al. [25] the near wall flow for for an open rim configuration was studied. The frequency analysis showed the same characteristic frequencies, found for the here investigated closed rim configuration. Hence, it can be concluded, that the characteristic frequencies found in both cases are related to the wheel/ wheelhouse interaction and not influenced by the rim design.

The described outflow of fluid on the upper wheel was attributed to cause the high pressure fluctuations. The derivation of this structure corresponds with findings from Regert \& Lajos [18]. In their work on a generic wheelhouse a dominating vortex was identified, which leaves the wheelhouse at the wheelhouse top and travels downstream along the side. It can be concluded that this structure is also present in the used full vehicle model and caused by the argumentations stated in their work.

\subsection{Completion of the flow field picture with CFD}

To complete the picture of the flow field CFD is used to visualize the 3D flow and the occurring flow conditions. The results are based on a simulation performed by Hobeika et. al [26], simulating a driving condition of $100 \mathrm{~km} / \mathrm{h}$ with zero yaw, using a hybrid RANS-LES turbulence modelling approach and with an Improved Delayed Detached Eddy Simulation (IDDES) formulation. Three seconds of physical 
time were computed, before the time averaged results over two seconds were calculated. The time step used was of $210^{-4} \mathrm{~s}$. The mesh consists of hexahedral cells with prism layers on the surface and resulting $y^{+}$values below 1 one on the exterior. The total mesh size was approximately 120 million cells. The detailed setup, as well as the results of a mesh and time step study can be found in Hobeika et al. [26]. Figure 11 shows the (time averaged) pressure distribution on the side of the vehicle. A comparison with the experimental pressure distribution, presented in Figure 4 show good agreement. The low pressure spot on the upper side od the wheel arch is captured in both methods, as well as the pressure recovery over the side. In the experiments the low pressure extends over a wider range compared to CFD, but this might be an interpolation error due to the corse measurement grid. Because of the side door and different safety beams it was not possible to have a finer grid in this area. Figure 11 shows also the wall shear stress lines, also called limiting streamlines, in the investigated area. Over the upper wheel arch edge, in the low pressure area, streamlines bent down over a short distance. Overall the limiting streamlines do not show any foci on the area and the flow seems to go downstream in almost horizontal direction.

Using the simulation allows to have a look in the 3D flow, in order to understand better the occurring structures. Figure 12 shows crossplanes at different $x / L$ locations. The height of the crossplanes reaches

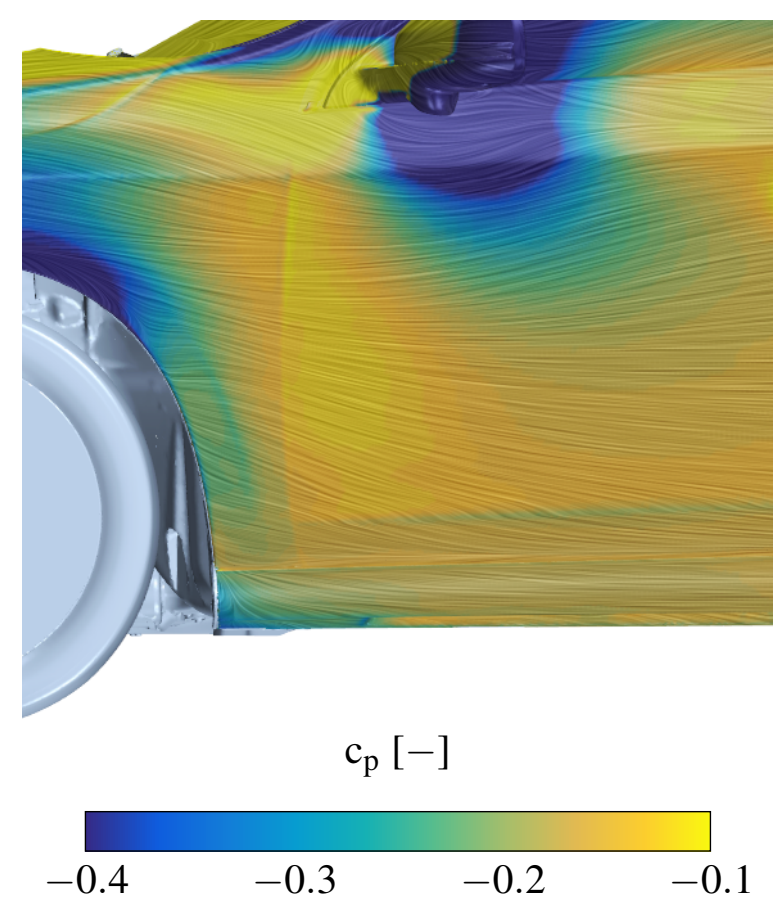

Fig. 11: Simulated pressure distribution behind the front wheel arch 
over the height covered in the experimental investigation. The planes are colored by the time averaged $\mathrm{X}$-vorticity component, where blue color shows negative $\mathrm{x}$-vorticity values and red positive ones. This gives also the information about the sense of rotation. As the $\mathrm{x}$ coordinate points in downstream direction, red is rotating positive and blue negative.

Figure $12 \mathrm{a}$ to $12 \mathrm{c}$ show that along the upper wheelhouse edge a structure is created with a negative sense of rotation. This means that flow exits from the wheelhouse and goes around the wheelhouse edge. This structure maintains also for the further downstream planes. At the lower edge a second structure with a negative sense of rotation can be observed. This is created further upstream and maintains downstream along the side of the car. These two structures can be interpreted as the ones identified by the cross correlation signals. Hence it can be seen that the unsteady pressure measurements are a suitable tool to identify flow phenomena occurring close to the surface.

\subsection{Results of the yaw configurations}

\subsubsection{Pressure distribution for yaw configurations}

For the following yaw configurations, the measurement area is located windward for negative angles and leeward for positive angles (see Figure 2). Figure 13 shows the standard deviation of the pressure coefficient for different yaw angles. The left column represents the windward and the right column the leeward orientation. As the investigated area under $\beta=-15^{\circ}$ is exposed to the oncoming wind, the standard deviations of the pressure are almost zero (Figure 13a). As the oncoming flow impinges the side under yaw, the flow is mostly attached. Fluctuations created by the wheel rotation and wheelhouse flow are suppressed compared to positive yaw angles (Figure 13a vs. Figure 13b). However, it seems that the strongest fluctuations are created along the upper wheelhouse edge shown by a remaining high fluctuation, measured in sensor 3, Figure 13a.

For positive yaw angles, the investigated area is oriented to leeward and the fluctuations increase with larger yaw angles. While the fluctuations along the lower wheelhouse edge increase only slightly, the high spot observed already in the zero yaw configuration, grows stronger and extends further downstream. 


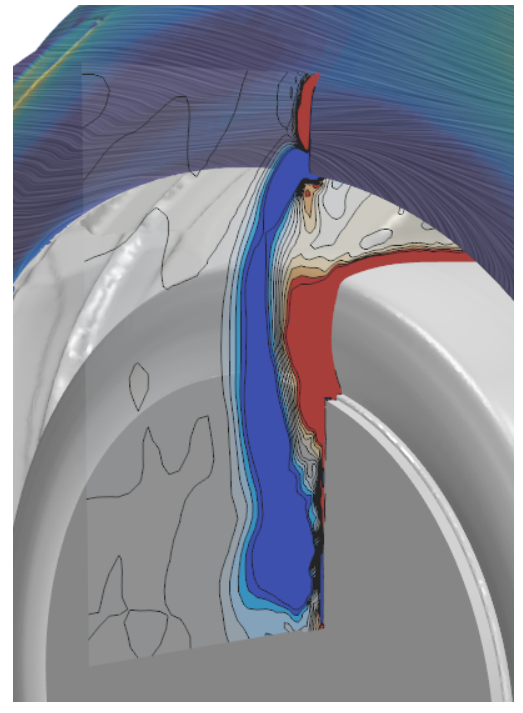

(a) $\frac{x}{L}=0.22$

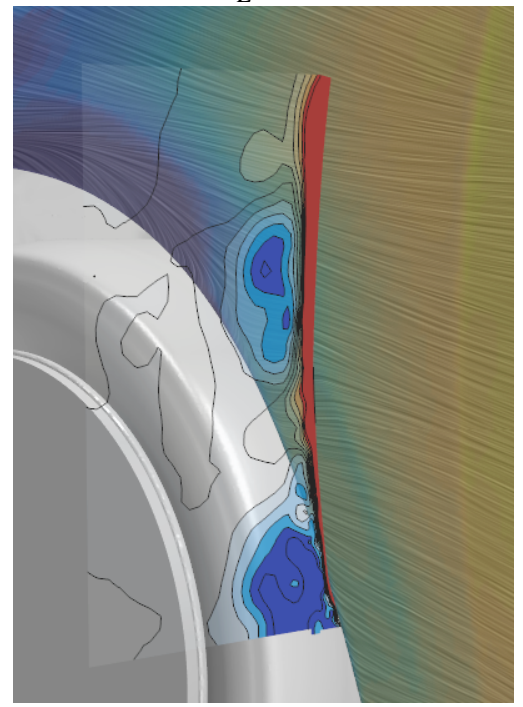

(d) $\frac{x}{L}=0.28$

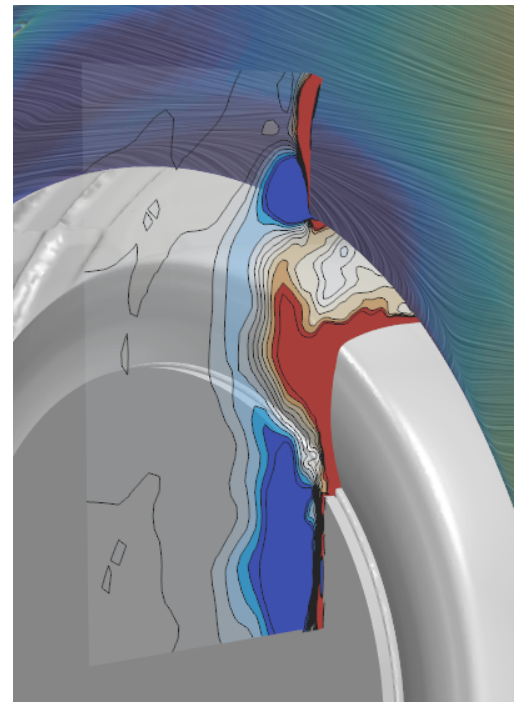

(b) $\frac{x}{L}=0.24$

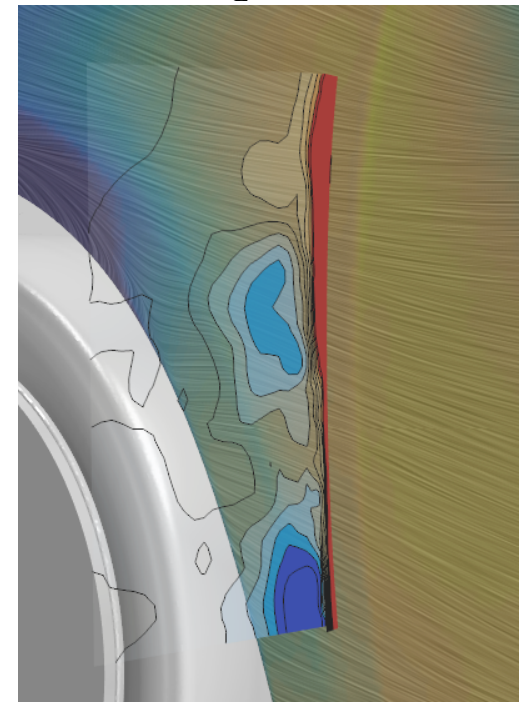

(e) $\frac{x}{L}=0.3$

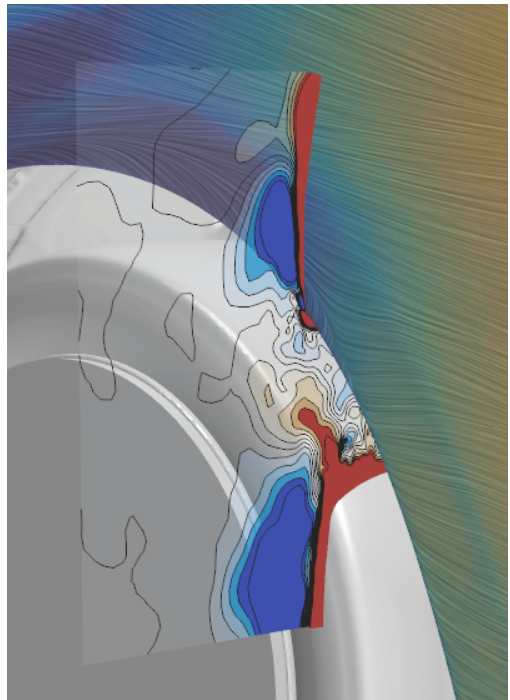

(c) $\frac{x}{L}=0.26$

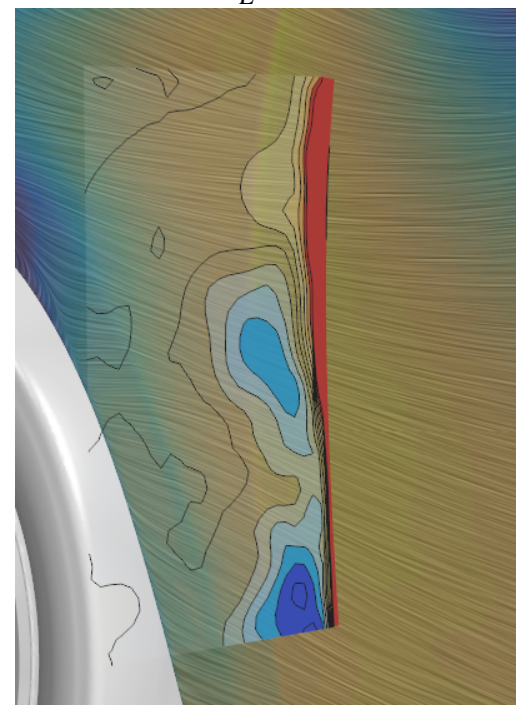

(f) $\frac{x}{L}=0.32$

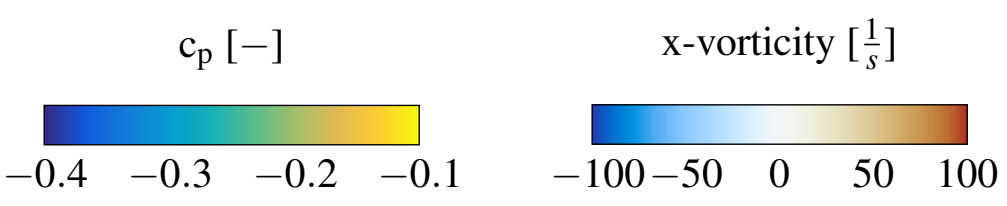

Fig. 12: Crossplanes colored by x-vorticity for different logitudinal distances; surface colored by $c_{p}$

\subsubsection{Frequency spectrum and cross correlation for yaw configurations}

For $\beta=+5^{\circ}, \beta=+10^{\circ}$ and $\beta=+15^{\circ}$, the dominant frequency at $13.9 \mathrm{~Hz}$ is present in all signals. The harmonics observed in the $\beta=0^{\circ}$ configuration are not present for $\beta=+15^{\circ}$ and partly present for $\beta=+10^{\circ}$ and $\beta=+5^{\circ}$. The peak at $1.9 \mathrm{~Hz}$ is not identifiable in the positive yaw configurations. The frequency spectrum of the negative yaw configurations shows clearly the $13.9 \mathrm{~Hz}$ and its harmonics. Also the peak at $1.9 \mathrm{~Hz}$ can be identified. For the $\beta=-10^{\circ}$ and $\beta=-15^{\circ}$ cases additional frequencies 
start to appear in the further downstream signals (approximately from sensor 20 onwards). The observed base frequency is $\mathrm{f}=14.9 \mathrm{~Hz}$ and occurs with its harmonics.

Figure 14 shows the cross correlations respective sensor 2 for different yaw angles. The distributions show that, regardless a leeward or windward orientation, the upper flow phenomena are restricted to the upper area. For negative yaw angles (windward) the structures are more limited in their spatial expansion compared to the positive yaw angle cases, meaning that the structures are suppressed by the oncoming flow.

\subsection{Discussion of the yaw configurations}

For the yaw configurations it was shown that the high fluctuations observed at zero yaw were reduced for negative yaw angles and amplified for positive yaw angles. As for negative angles the measurement

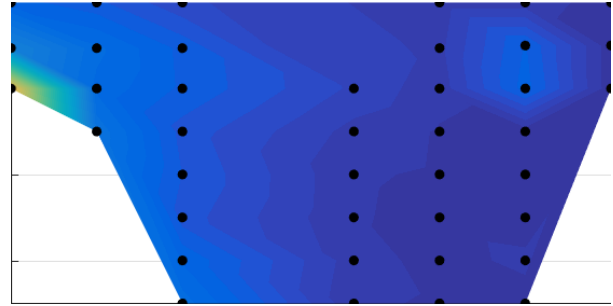

(a) $\operatorname{std} c_{p}, \beta=-15^{\circ}$

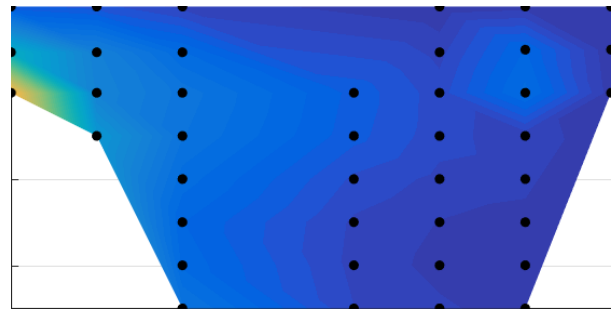

(c) $\operatorname{std} c_{p}, \beta=-10^{\circ}$

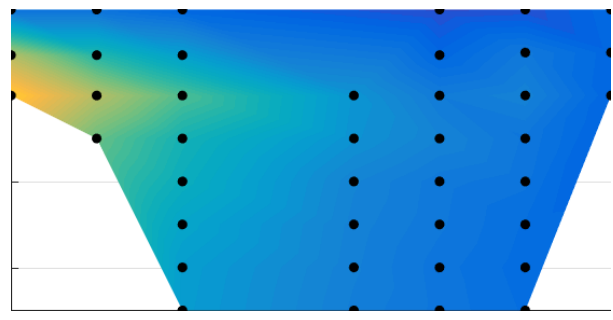

(e) $\operatorname{std} c_{p}, \beta=-5^{\circ}$

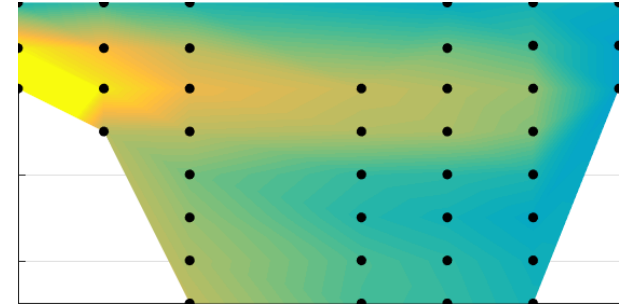

(b) $\operatorname{std} c_{p}, \beta=+15^{\circ}$

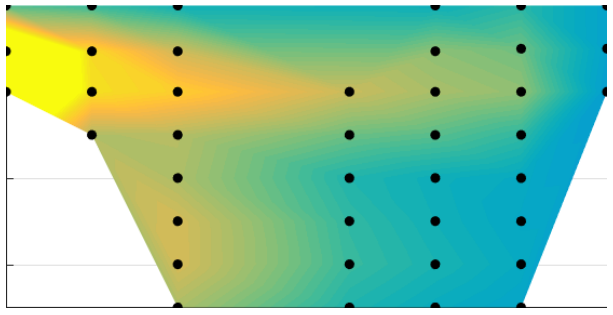

(d) $\operatorname{std} c_{p}, \beta=+10^{\circ}$

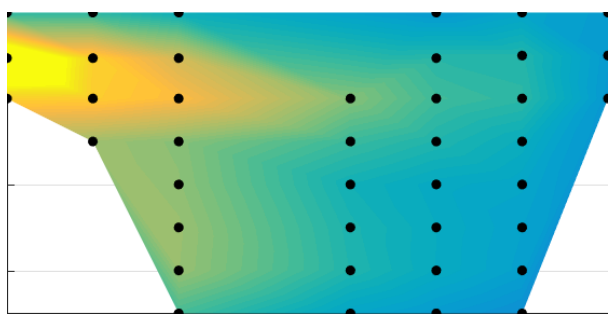

(f) $\operatorname{std} c_{p}, \beta=+5^{\circ}$

$$
\begin{array}{llll}
\multicolumn{4}{c}{\text { std } c_{\mathrm{p}}[-]} \\
\\
\hline 0.00 & 0.05 & 0.10 & 0.15
\end{array}
$$

Fig. 13: Standard deviation of the pressure coefficient for different yaw angles 


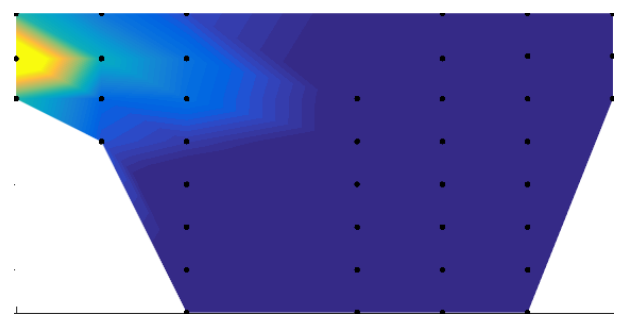

(a) $\beta=-15^{\circ}$

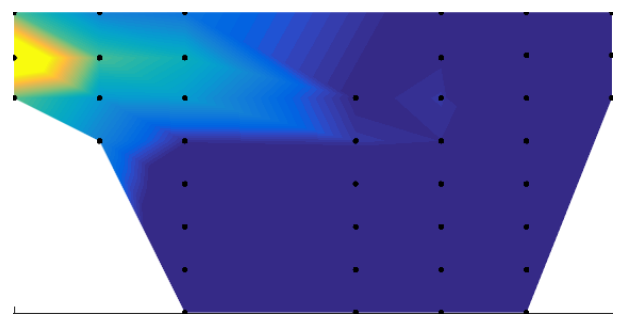

(c) $\beta=-10^{\circ}$

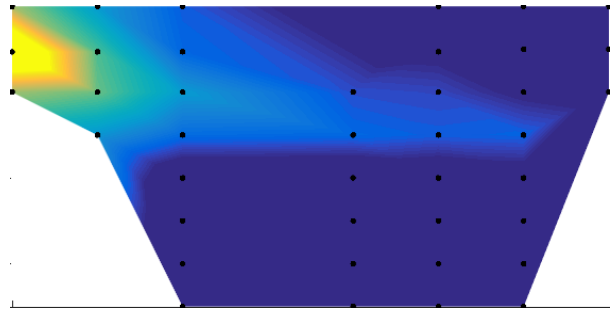

(e) $\beta=-5^{\circ}$

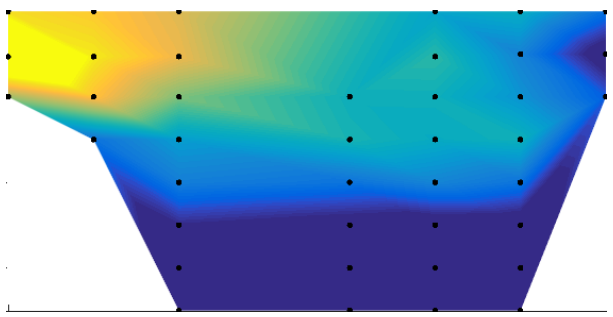

(b) $\beta=+15^{\circ}$

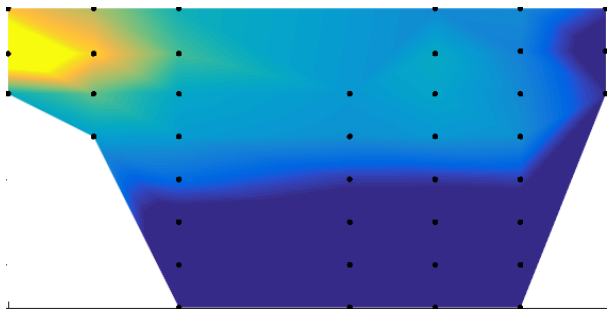

(d) $\beta=+10^{\circ}$

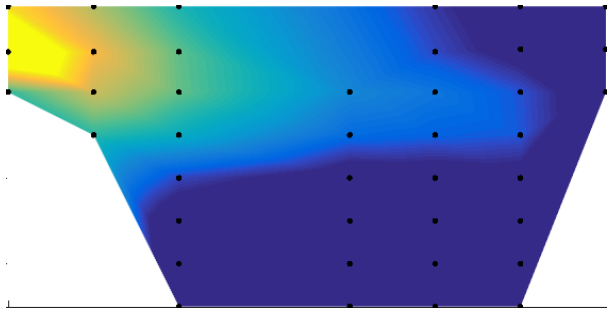

(f) $\beta=+5^{\circ}$

correlation coefficient

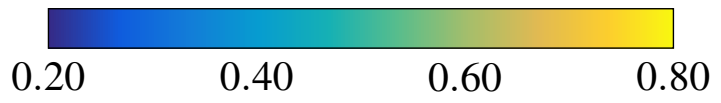

Fig. 14: Cross correlations for different yaw angles respective sensor 2

area was oriented windward, the flow structure exiting the wheelhouse is suppressed in its downstream expansion. For the positive yaw angles, the investigated area is orientated to leeward and therefore also in the separation area created by the car front. Additionally, the flow underneath the car exits at the side with a larger angle than under zero yaw. Hence, the wheel-wheelhouse flow interaction is stronger and the flow leaving the wheelhouse on the upper side of the wheel is less suppressed by the high momentum flow around the car. However, the structure exiting the wheel house remains, even under large yaw angles and plays therefore a significant roll in the different configurations. In the discussion for the zero yaw case it was mentioned, that this main structure can be related to a vortex exiting the wheel house, as found by Regert \& Lajos [18]. The observation that the resulting pressure fluctuations are still significant under yaw conditions show the dominance of this vortex. This is especially important in connection with 
side contamination, as this structure will contribute to it in all investigated yaw conditions. Further it can be noted that the propagation direction does not change with different yaw angles and is in all cases directed horizontally downstream. More desirable would be a downwards oriented propagation to lead the contaminants away from the side doors.

Regarding the frequency analysis, the flow field under yaw conditions showed throughout a yaw range of $\beta=-15^{\circ}$ to $\beta=+15^{\circ}$ a dominant frequency of $\mathrm{St}=0.2$. The $\mathrm{St}=0.03$ peak was only observed for the negative yaw configurations. For the negative yaw angles the flow was overall more attached than for positive yaw angles as the measurement area was located windward. The reduced separation also seemed to reduce the noise in the pressure signals and might therefore be the reason why the frequencies are clearer observable. Especially the low frequency at $\mathrm{St}=0.03$ seems to be more pronounced under these conditions, while it is buried in the positive yaw configurations.

Interesting is the occurrence of another frequency at $14.9 \mathrm{~Hz}$, which was observed for $\beta=-15^{\circ}$. It seems that due to the high yaw angle a kind of bi-frequency structure was created, which occurs at $13.9 \mathrm{~Hz}$ and $14.9 \mathrm{~Hz}$.

\section{Conclusion}

Experimental investigations downstream of the front wheel of a full-scale passenger car were conducted to understand the occurring flow structures due to the wheel-wheelhouse interaction and how they were changed under different yaw conditions. Unsteady surface pressure measurements were taken downstream of the front wheel arch to study the near wall flow based on pressure distribution, standard deviation, characteristic frequencies and cross correlations.

The frequency analysis showed dominant frequencies at $\mathrm{f}=1.9 \mathrm{~Hz}(\mathrm{St}=0.03)$ and $13.9 \mathrm{~Hz}(\mathrm{St}=0.2)$. The lower value is related to a flow interaction with the wheelhouse geometry.

A Strouhal number of $\mathrm{St}=0.2$ is a characteristic number found for various separation phenomena. It is assumed that this frequency can be related to a pumping effect from the flow exiting the wheel house. The measurement with stationary wheels showed that the dominant frequency at St $=0.2$ and its harmonics diminished. However, the peak at $\mathrm{St}=0.03$ was still present. Therefore, it can be concluded that the most dominant frequency of $\mathrm{St}=0.2$ is related to a separation phenomena created by the wheel rotation and its interaction with the wheelhouse flow, while $\mathrm{St}=0.03$ is related to an interaction with 
the wheelhouse geometry independently from the wheel rotation. A similar characteristic frequency was also found in literature for measurements downstream of a rear wheelhouse. A comparison with frequencies found in a study with an open rim design, showed that the same characteristic frequencies were found as in this study. This shows that they are independent from the rim design.

The analysis of the cross correlation signals showed that a larger coherent structure is present in the upper part behind the wheel house, which is independent from the flow in the lower part. This structure reaches approximately over the area, where also the high pressure standard deviations were observed. The occurrence of a vortex exiting the top wheel house and resulting in a downstream travelling vortex was also found in an investigation on a generic wheelhouse model and it can therefore be concluded that in this investigation the same phenomena is observed by means of pressure measurements. In the lower part no large coherent structure was observed. The correlation signals showed only very weak similarities to the neighbouring signals. Further downstream, the flow travels almost undisturbed in horizontal direction. The propagation speed was estimated to be approx. $14 \mathrm{~m} / \mathrm{s}$.

The yaw configurations showed more pronounced frequency signals when the measurement area was located windward. Especially the $\mathrm{St}=0.03$ peak is clearer and seemed to be buried in noise for the positive yaw configurations. For $\beta=-15^{\circ}$ a bi-frequency phenomenon was observed. Which showed dominant frequencies at $13.9 \mathrm{~Hz}$ and $14.9 \mathrm{~Hz}$.

The structure exiting the wheelhouse was related to a vortex, which was also identified on a generic model and is present for all yaw conditions. This shows its strength, dominance and general occurrence, and has therefore to be considered in the design process as this causes most of the side contamination. Analysing the pressure signals allows to determine the propagation direction, which has to be taken into account, as to reduce the soiling, a more downwards directed propagation is desired, to be able to lead the contaminants away from critical areas.

\section{Acknowledgements}

We would like to thank Volvo Car Corporation for their support and use of their resources. A special thanks goes to Alexander Broniewicz for his contributions and help throughout the work.

We also would like to thank the Swedish Energy Agency and the Strategic Vehicle Research and Innovation for funding this project. 


\section{References}

[1] Gulyás, A., Bodor, Á., Regert, T., and Jánosi, I. M., 2013. "Piv measurement of the flow past a generic car body with wheels at les applicable reynolds number". International Journal of Heat and Fluid Flow, 43, pp. 220-232.

[2] Bruneau, C.-H., Creusé, E., Gilliéron, P., and Mortazavi, I., 2014. "Effect of the vortex dynamics on the drag coefficient of a square back ahmed body: Application to the flow control". European Journal of Mechanics - B/Fluids, 45, pp. 1-11.

[3] Rossitto, G., Sicot, C., Ferrand, V., Borée, J., and Harambat, F., 2016. "Influence of afterbody rounding on the pressure distribution over a fastback vehicle". Experiments in Fluids, 57(3).

[4] Meile, W., Ladinek, T., Brenn, G., Reppenhagen, A., and Fuchs, A., 2016. "Non-symmetric bistable flow around the ahmed body". International Journal of Heat and Fluid Flow, 57(Supplement C), pp. 34-47.

[5] Landström, C., 2011. "Investigation of aerodynamic wheel designs on a passenger car at different cooling air flow conditions". In Proceedings of ASME-JSME-KSME Joint Fluids Engineering Conference. Hamatsu, Shizuoka, Japan.

[6] Hobeika, T., Gullberg, P., Sebben, S., and Lofdahl, L., 2017. "Force based measurement method for cooling flow quantification”. SAE International Journal of Passenger Cars - Mechanical Systems, $10(2)$.

[7] Wäschle, A., 2007. "The influence of rotating wheels on vehicle aerodynamics - numerical and experimental investigations". In SAE World Congress \& Exhibition, SAE Technical Paper Series, SAE International.

[8] Zhiling, Q., Landström, C., Löfdahl, L., and Josefsson, L., 2010. "Wheel aerodynamic developments on passenger cars by module-based prototype rims and stationary rim shields". In FISITA 2010 Automotive World Congress.

[9] Landstrom, C., Josefsson, L., Walker, T., and Lofdahl, L., 2012. "Aerodynamic effects of different tire models on a sedan type passenger car". SAE International Journal of Passenger Cars Mechanical Systems, 5(1), pp. 136-151. 
[10] Landström, C., Walker, T., Christoffersen, L., and Löfdahl, L., 2011. "Influences of different front and rear wheel designs on aerodynamic drag of a sedan type passenger car". In SAE World Congress \& Exhibition, SAE Technical Paper Series, SAE International400 Commonwealth Drive, Warrendale, PA, United States.

[11] Landström, C., Josefsson, L., Walker, T., and Löfdahl, L., 2011. "An experimental investigation of wheel design parameters with respect to aerodynamic drag”. In FKFS Conference Paper, Vol. 10, pp. 5-6.

[12] Hobeika, T., Sebben, S., and Landstrom, C., 2013. "Investigation of the influence of tyre geometry on the aerodynamics of passenger cars". SAE International Journal of Passenger Cars Mechanical Systems, 6(1), pp. 316-325.

[13] Schnepf, B., Schütz, T., and Indinger, T., 2015. "Further investigations on the flow around a rotating, isolated wheel with detailed tread pattern”. SAE International Journal of Passenger Cars - Mechanical Systems, 8(1).

[14] Axon, L., Garry, K., and Howell, J., 1998. "An evaluation of cfd for modelling the flow around stationary and rotating isolated wheels". In SAE World Congress \& Exhibition, SAE Technical Paper Series, SAE International400 Commonwealth Drive, Warrendale, PA, United States.

[15] Dimitriou, I., and Klussmann, S., 2006. "Aerodynamic forces of exposed and enclosed rotating wheels as an example of the synergy in the development of racing and passenger cars". In SAE World Congress \& Exhibition, SAE Technical Paper Series, SAE International400 Commonwealth Drive, Warrendale, PA, United States.

[16] Emma Croner, Hervé Bézard, Christophe Sicot, and Guillaume Mothay, 2013. “Aerodynamic characterization of the wake of an isolated rolling wheel”. International Journal of Heat and Fluid Flow(0), pp. -

[17] Landström, C., Löfdahl, L., and Walker, T., 2009. "Detailed flow studies in close proximity of rotating wheels on a passenger car". SAE International Journal of Passenger Cars - Mechanical Systems, 2(1), pp. 861-874.

[18] Tamas Regert, and Tamas Lajos, 2007. "Description of flow field in the wheelhouses of cars". International Journal of Heat and Fluid Flow, 28(4), pp. 616-629.

[19] Söderblom, D., 2012. Wheel housing aerodynamics of heavy trucks, Vol. ny ser., nr. 3309 of Dok- 
torsavhandlingar vid Chalmers tekniska högskola. Chalmers University of Technology, Göteborg.

[20] Bonitz, S., Larsson, L., Lofdahl, L., and Broniewicz, A., 2015. "Structures of flow separation on a passenger car”. SAE International Journal of Passenger Cars - Mechanical Systems, 8(1).

[21] Gaylard, A. P., and Duncan, B., 2011. "Simulation of rear glass and body side vehicle soiling by road sprays". SAE International Journal of Passenger Cars - Mechanical Systems, 4(1), pp. 184196.

[22] Gaylard, A. P., Kirwan, K., and Lockerby, D. A., 2017. "Surface contamination of cars: A review". Proceedings of the Institution of Mechanical Engineers, Part D: Journal of Automobile Engineering, 231(9), pp. 1160-1176.

[23] Sternéus, J., Walker, T., and Bender, T., 2007. Upgrade of the Volvo Cars Aerodynamic Wind Tunnel. SAE International, Warrendale, PA.

[24] Wieser, D., Schmidt, H.-J., Müller, S., Strangfeld, C., Nayeri, C., and Paschereit, C., 2014. "Experimental comparison of the aerodynamic behavior of fastback and notchback drivaer models". In SAE 2014 World Congress and Exhibition. SAE International, Warrendale, PA.

[25] Bonitz, S., Wieser, D., Broniewicz, A., Larsson, L., Lofdahl, L., Nayeri, C., and Paschereit, C., 2018. "Experimental investigation of the near wall flow downstream of a passenger car wheel arch”. SAE International Journal of Passenger Cars - Mechanical Systems, 11(1).

[26] Hobeika, T., and Sebben, S., 2018. "Cfd investigation on wheel rotation modelling”. Journal of Wind Engineering and Industrial Aerodynamics, 174, pp. 241 - 251. 